\title{
Experimental Demonstration of a Classical Analog to Quantum Noise Cancellation for Use in Gravitational Wave Detection
}

\author{
C. M. Mow-Lowry, B. S. Sheard, M. B. Gray, and D. E. McClelland \\ Gravitational Wave Research Facility, Department of Physics, Faculty of Science, The Australian National University, \\ ACT 0200, Australia \\ S. E. Whitcomb \\ LIGO Laboratory, California Institute of Technology, Pasadena, California, 91125, USA
}

(Received 13 October 2003; published 23 April 2004)

\begin{abstract}
We present results that are a classical analog to quantum noise cancellation. It is possible to breach the standard quantum limit in an interferometer by the use of squeezing to correlate orthogonal quadratures of quantum noise, causing their effects on the resulting sensitivity to cancel. A laser beam incident on a Fabry-Perot cavity was imprinted with classical, correlated noise in the same quadratures that cause shot noise and radiation pressure noise. Couplings between these quadratures due to a movable mirror, sensitive to radiation pressure, cause the excess classical noise to cancel. This cancellation was shown to improve the signal to noise ratio of an injected signal by approximately a factor of 10 .
\end{abstract}

DOI: 10.1103/PhysRevLett.92.161102

PACS numbers: 04.80.Nn, 07.60.Ly, 42.50.Lc

The quest to detect gravitational waves is pushing the sensitivity of interferometric measurements to their quantum limits. It is expected that a decade from now gravitational wave detectors will be limited in sensitivity by quantum fluctuations of the optical fields used to measure the position of test masses. They will operate at the standard quantum limit (SQL) where shot noise in the detected light and radiation pressure noise on the mirrors cause equal errors in the position measurement $[1,2]$. There have been several techniques proposed to breach the SQL. These include a possible change of readout parameter [3], the use of different configurations to take advantage of optomechanical couplings [4], quantum locking $[5,6]$, and the use of squeezing to reduce quantum noise $[7,8]$.

In recent times, squeezing has attracted the most attention. This is due to the relative experimental maturity of squeezing $[9,10]$ and its potential for incorporation into a gravitational wave detector [8]. In a coherent state, the quantum fluctuations of phase and amplitude are equal and uncorrelated. Squeezing alters these noise characteristics, making the noise in one quadrature smaller, at the expense of the noise in the orthogonal quadrature. Recently it was experimentally demonstrated that squeezing can be used to improve the sensitivity of a shot noise limited power recycled Michelson interferometer [11].

The use of squeezing to breach the SQL has not yet been demonstrated. In fact, despite intensive and increasing effort [12-15], no one has built an optomechanical device that even reaches the SQL. However, we demonstrate that it is possible to experimentally verify the physics behind the use of squeezed light to breach the
SQL by using a classical analog of quantum noise reduction. Moreover, our experiment shows that squeezed light interferometry can be understood in purely classical terms, demystifying quantum physics. This could aid in designing new ways of beating the SQL and potentially lead to a better understanding of other quantum optical devices.

In an interferometer the positions of test masses (mirrors) are measured using the phase of an optical field. As such, phase fluctuations in this field couple directly to the output. If the mirrors are movable, amplitude fluctuations change the positions of the mirrors via the radiation pressure force. Thus, amplitude fluctuations are coupled into the orthogonal phase quadrature and also effect the total sensitivity. Squeezing can be used to reduce the fluctuations in one quadrature only. The "squeeze angle," relative to the phase of the light in the interferometer, determines which quadrature has reduced fluctuations.

Phase measurements at the SQL are limited equally by radiation pressure noise (rpn) and shot noise (sn). The instantaneous noise has two terms:

$$
\phi_{\mathrm{tot}}=\phi_{\mathrm{sn}}+\phi_{\mathrm{rpn}}
$$

which gives an rms output, and

$$
\sqrt{\left\langle\phi_{\mathrm{tot}}^{2}\right\rangle}=\sqrt{\left\langle\phi_{\mathrm{sn}}^{2}\right\rangle+\left\langle\phi_{\mathrm{rpn}}^{2}\right\rangle+2\left\langle\phi_{\mathrm{sn}} \phi_{\mathrm{rpn}}\right\rangle}
$$

where $\phi_{\mathrm{rpn}}$ is determined by the radiation pressure force, the mechanical response of the mirror, and the interferometer response to a given length change. The final term in Eq. (2) is an interference term. Since shot noise and radiation pressure noise are uncorrelated in a coherent state, this term averages to zero, while the remaining 
terms add in quadrature to form the standard quantum limit. However, if the quantum noise is squeezed with a $45^{\circ}$ angle, as shown in Fig. 1(b), the noise in the amplitude and phase quadratures is correlated. Despite increasing both shot noise and radiation pressure noise, the correlation generates a nonzero interference term which can reduce the total noise below the standard quantum limit.

In the classical analog of quantum noise cancellation, quantum noise fluctuations were replaced by classical modulations. These modulations were imprinted on a laser beam via an amplitude modulator and a frequency modulator. Driving the modulators with uncorrelated noise of the same amplitude generates a classical analog to the coherent state, with much higher noise in all quadratures. If both the modulators are driven by the same noise source, the noise in the amplitude and phase quadratures will be correlated.

Quantum noise cancellation requires the measurement of tiny fluctuations. However, for classical noise cancellation the size of the modulations can be orders of magnitude larger than the quantum equivalents. For this reason, classical noise cancellation can be observed in a much noisier environment. Also, the modulations can be arbitrarily correlated without the need of low frequency squeezing. These changes make it possible to demonstrate the principle of noise cancellation in a bench-top environment.

The key to this experiment was an interferometer sensitive to radiation pressure. This was a Fabry-Perot cavity in which one mirror was mounted on a niobium cantilever flexure. A force on the mirror bends the cantilever, linearly extending the length of the cavity, for small motion. The mechanical oscillator had a resonance frequency of $303 \mathrm{~Hz}$, a quality factor of order 3000, a mass of approximately $1.2 \mathrm{~g}$, an angular spring constant of $0.45 \mathrm{Nm} / \mathrm{rad}$, and a lever arm of $12.7 \mathrm{~mm}$.

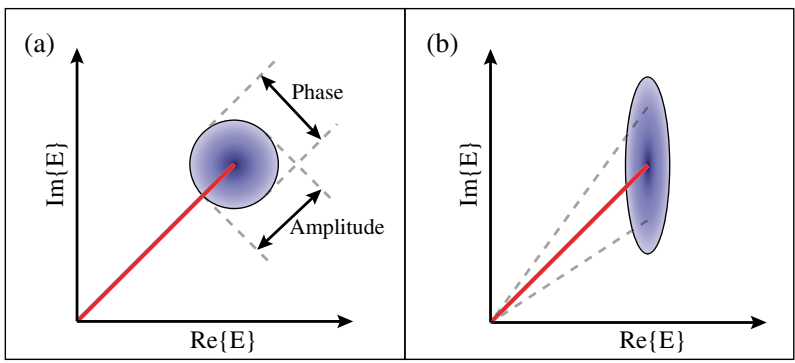

FIG. 1 (color online). (a) shows a coherent state, where a circular ball of quantum noise is superimposed on an unchanging coherent amplitude, or "stick." (b) shows squeezing at a $45^{\circ}$ angle. This correlates the noise in the amplitude and phase quadratures; i.e., amplitude increase is correlated with phase advance and vice versa. This is demonstrated by the dotted lines.
The circulating power in the cavity was proportional to the incident power. As such, amplitude modulation changed the circulating power in the cavity, and hence the radiation pressure force on the movable mirror. Because of the rapid response of the cavity (100 ns time constant) compared with the mechanical oscillator, dynamic effects of the cavity were negligible. The requisite amplitude modulation was generated using a common mirror Michelson interferometer, shown in the dotted box in Fig. 2. Controlled modulation of the position of this mirror, using a piezoelectric transducer (PZT), resulted in amplitude modulation of the output field with no corresponding phase change. Frequency modulation was provided by stressing the laser crystal with a PZT. This technique generated no measurable amplitude modulation.

Two orthogonally polarized beams were incident on the test cavity: a high power pump and a low power probe. Both beams were frequency modulated by the laser, but only the high power pump beam was amplitude modulated by the Michelson interferometer. At the cavity transmitted port the pump beam was rejected to better than one part in $10^{4}$ using a Glan-Taylor polarizer. As such only the probe beam was incident on the transmitted port photodetector. This arrangement reduced mixing between the amplitude modulation and the cavity error signal readout. Figure 2 is a schematic diagram of the optical layout.

The test cavity length was monitored and controlled using rf transmission locking [16]. The rf phase modulation sidebands were imposed only on the probe beam at $19 \mathrm{MHz}$. The signal on the transmitted detector was demodulated at $19 \mathrm{MHz}$, creating an error signal which was then used to control the position of the static mirror in the cavity via a PZT. The unity gain frequency of the cavity length feedback was approximately $0.5 \mathrm{~Hz}$. Higher frequency feedback was attenuated by a third order elliptic filter with a corner frequency of $10 \mathrm{~Hz}$. Thus, the error

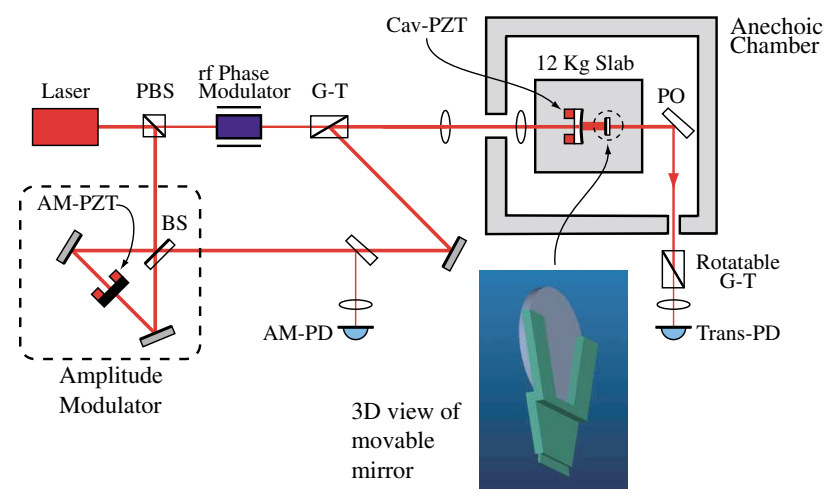

FIG. 2 (color online). A schematic diagram of the optical layout. A close-up view of the flexure-mirror oscillator is also shown. PBS, polarizing beam splitter; BS, beam splitter; G-T, Glan-Taylor polarizer; PO, pickoff mirror; PD, photodetector. 
signal provided an out of loop measurement of the cavity length for frequencies higher than $20 \mathrm{~Hz}$.

To generate an accurate analog to quantum noise cancellation, it was important to ensure that the flexure mirror was being driven by the radiation pressure force. The transfer function from the amplitude modulator drive to the error signal was analyzed to determine whether radiation pressure was the dominant cause of error signal disturbance. The error signal response was well modeled by including three major effects. The first was an unfitted calculation of the effect of the radiation pressure force on the error signal. The second was a thermally induced length change caused by heating of the mirror coatings. The third was spurious coupling between amplitude modulation and the error signal. The cross polarized pump and probe configuration was used to minimize the third effect. Figure 3 shows the experimental transfer function plotted with the combined model and the unfitted radiation pressure component of that model. From the model it was determined that the radiation pressure force induced motion was the dominant cause of error signal disturbance resulting from amplitude modulation for frequencies between $100 \mathrm{~Hz}$ and $1 \mathrm{kHz}$. Further details of this model, including a description of the phase component of the transfer function, are presented in Ref. [17].

To record noise cancellation, broadband white noise was injected into both the amplitude and frequency modulators. By adjusting the relative gains of the modulators it was possible to create an environment where the error signal disturbance from each modulation was equal. This was analogous to injecting a strongly amplitude or
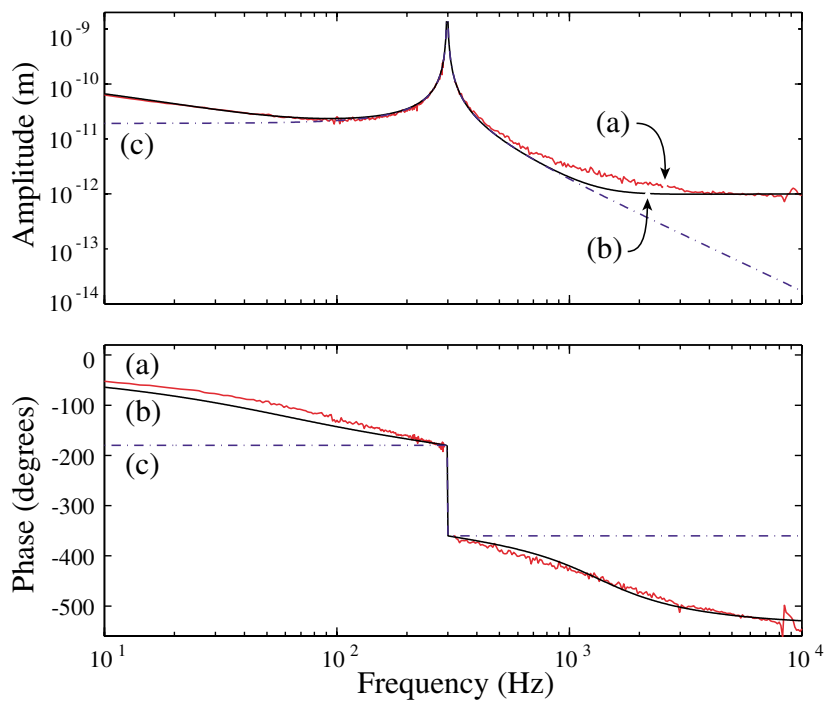

FIG. 3 (color online). This plot shows the error signal response from incident amplitude modulation. Trace $(a)$ is the experimental data, trace $(b)$ is the combined model, and trace $(c)$ is the unfitted radiation pressure component of that model. phase squeezed beam into a quantum noise limited interferometer, since the sensitivity was limited only by the noisy quadrature. By driving both modulators with the same signal, they became very well correlated. This was analogous to rotating the squeeze angle from amplitude (or phase) squeezing to $45^{\circ}$.

The effective squeeze angle was adjusted by giving the noise used to drive one of the modulators a frequency independent variable phase shift. Adjustment of this phase shift caused the two noise sources to add constructively or destructively. The magnitude of the drive to one modulator was adjusted to allow for the different responses between modulator drives and error signal disturbances. To achieve broadband cancellation across the full spectrum, a filter matched to the mechanical response of the flexure-mirror oscillator was required. Such a filter was unavailable, so cancellation was restricted to a smaller bandwidth. The particular frequency band for noise cancellation was chosen based on the response of the error signal to amplitude modulation: (i) it must be dominated by radiation pressure, and (ii) the response must be nearly flat.

To satisfy these criteria, based on the response shown in Fig. 3, the frequency band from 150 to $200 \mathrm{~Hz}$ was selected. Band-limited white noise was generated and used to drive the two modulators. The drive for the frequency modulator was then shifted in phase and passed through a filter to match the response of the amplitude modulator in the error signal. After finetuning, the frequency and amplitude modulators had approximately the same effect on the error signal, and it was then possible to inject both modulations creating the optimal correlations for cancellation. The sign of the frequency modulation drive was then flipped to achieve either constructive (not shown) or destructive interference. To demonstrate that this procedure canceled the noise without affecting the sensitivity to valid signals, a signal was added through the cavity length control PZT (an independent actuator) to demonstrate an improvement in the signal to noise ratio.

Figure 4 shows several traces, all of which are noise spectra of the cavity error signal. Trace $(d)$ is the background noise, recorded with no optical modulations added in the frequency band shown. The spike, at $176 \mathrm{~Hz}$, was the signal injected through the cavity PZT. Traces $(a)$ and $(b)$ show the excess noise added first through the amplitude modulator and second through the frequency modulator. Trace $(c)$ shows the noise, originating from the two orthogonal quadratures, canceling to give a quieter resultant. The improvement in the signal to noise ratio was approximately a factor of 10 .

The experiment presented here was a classical analog to a quantum system. The results shown comprise a proof of principle for noise cancellation, a technique proposed as a means of breaching the standard quantum limit. The experiment also allowed for development of expertise 


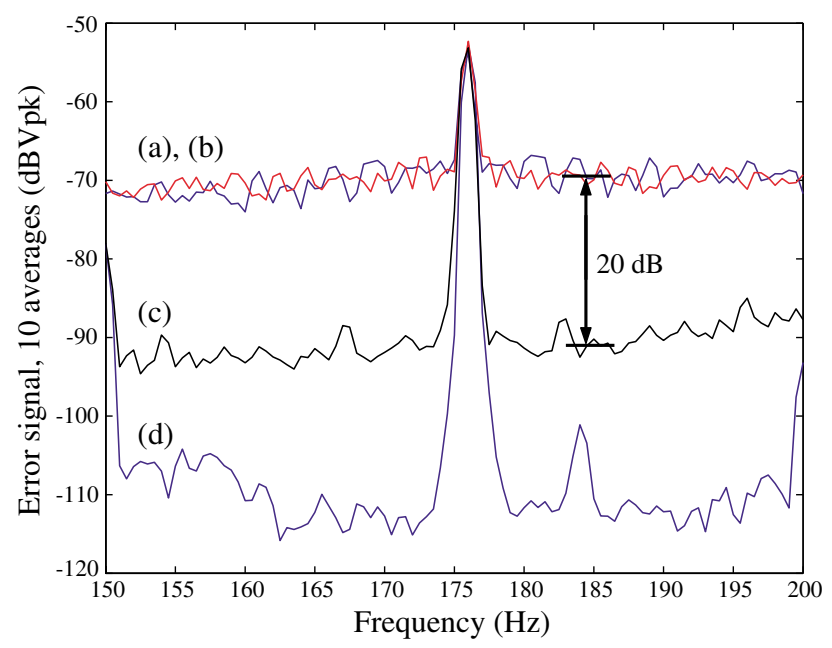

FIG. 4 (color online). When amplitude and frequency noise are present and correlated, the total sensitivity is $20 \mathrm{~dB}$ better than with only one source of noise present.

with radiation pressure dominated systems, an important step toward reaching, and eventually breaching, the standard quantum limit.

The authors wish to thank $\mathrm{Li}$ Ju for her assistance and advice in constructing the niobium flexure and Daniel Shaddock for his significant creative contributions. This research was completed under the auspices of ACIGA supported by the Australian Research Council. This material was supported in part by the United States National Science Foundation under Cooperative Agreement No. PHY-0107417. This paper has been assigned LIGO Laboratory Document No. LIGO-P030051-00-R.
[1] C. M. Caves, K. S. Thorne, R.W. P. Drever, V. D. Sandberg, and M. Zimmermann, Rev. Mod. Phys. 52, 341 (1980).

[2] C. M. Caves, Phys. Rev. D 23, 1693 (1981).

[3] V. B. Braginsky, Y. I. Vorontsov, and K. Thorne, Science 209, 547 (1980).

[4] A. Buonanno and Y. Chen, Phys. Rev. D 64, 042006 (2000).

[5] J.-M. Courty, A. Heidmann, and M. Pinard, Phys. Rev. Lett. 90, 083601 (2003).

[6] J.-M. Courty, A. Heidmann, and M. Pinard, Europhys. Lett. 63, 226 (2003).

[7] W.G. Unruh, in Quantum Optics, Experimental Gravitation and Measurement Theory, edited by P. Meystre and M. O. Scully (Plenum, New York, 1983), p. 647.

[8] H. J. Kimble, Y. Levin, A. B. Matsko, K. S. Thorne, and S. P. Vyatchanin, Phys. Rev. D 65, 022002 (2001).

[9] K. Schneider, M. Lang, J. Mlynek, and S. Schiller, Opt. Express 2, 59 (1998).

[10] P. K. Lam, T. C. Ralph, B. C. Buchler, D. E. McClelland, H.-A. Bachor, and J. Gao, J. Opt. B 1, 469 (1999).

[11] K. McKenzie, D. A. Shaddock, D. E. McClelland, B. C. Buchler, and P. K. Lam, Phys. Rev. Lett. 88, 231102 (2002).

[12] I. Tittonen et al., Phys. Rev. A 59, 1038 (1999).

[13] P. F. Cohadon, A. Heidmann, and M. Pinard, Phys. Rev. Lett. 83, 3174 (1999).

[14] P. K. Lam (personal communication).

[15] N. Mavalvala (personal communication).

[16] A. D. White, IEEE J. Quantum Electron. 1, 349 (1965).

[17] C. M. Mow-Lowry, B. S. Sheard, S. E. Whitcomb, M. B. Gray, and D. E. McClelland (unpublished). 